RESEARCH PAPER

\title{
Impact of the graphic Canadian warning labels on adult smoking behaviour
}

\author{
D Hammond, G T Fong, P W McDonald, R Cameron, K S Brown
}

Tobacco Control 2003;12:391-395

See end of article for authors' affiliations avthors afiliations

Correspondence to: David Hammond, MSc, Department of Psychology, University of Waterloo, 200 University Avenue West, Waterloo, Ontario N21 3G1, Canada; dhammond@ uwaterloo.ca

Received 15 April 2003 Accepted 21 August 2003

\begin{abstract}
Objective: To assess the impact of graphic Canadian cigarette warning labels on current adult smokers. Design: A random-digit-dial telephone survey was conducted with 616 adult smokers in south western Ontario, Canada in October/November 2001, with three month follow up.

Main outcome measures: Smoking behaviour (quitting, quit attempts, and reduced smoking), intentions to quit, and salience of the warning labels.

Results: Virtually all smokers (91\%) reported having read the warning labels and smokers demonstrated a thorough knowledge of their content. A strong positive relation was observed between a measure of cognitive processing - the extent to which smokers reported reading, thinking about, and discussing the new labels - and smokers' intentions to quit (odds ratio (OR) 1.11,95\% confidence interval (CI) 1.07 to $1.16 ; p<0.001$ ). Most important, cognitive processing predicted cessation behaviour at follow up. Smokers who had read, thought about, and discussed the new labels at baseline were more likely to have quit, made a quit attempt, or reduced their smoking three months later, after adjusting for intentions to quit and smoking status at baseline (OR $1.07,95 \% \mathrm{Cl} 1.03$ to $1.12 ; \mathrm{p}<0.001$ ).

Conclusions: Graphic cigarette warning labels serve as an effective population based smoking cessation intervention. The findings add to the growing literature on health warnings and provide strong support for the effectiveness of Canada's tobacco labelling policy.
\end{abstract}

$\mathrm{T}$ obacco use is the leading cause of premature mortality in the developed world. ${ }^{1}$ Cigarette warning labels are one of several national level policies that have been introduced to address the economic and health burden of tobacco use. However, warning labels are unique among tobacco control initiatives in that they are delivered at the time of smoking. As a result, virtually all smokers are exposed to the intervention, and pack-a-day smokers are potentially exposed to the warnings over 7000 times per year.

Given this reach and frequency of exposure, even if warning labels have only a modest effect size on individual smokers they may have a dramatic impact upon smoking behaviour at the population level.

Research has identified the basic principles for enhancing the effectiveness of tobacco warning labels: colour pictures or graphics, positioning on the front of packs, increases in size, and direct unambiguous messages all increase the likelihood that smokers will notice warnings labels. ${ }^{2}$ However, because of the pragmatic challenges of evaluating population based policy initiatives, field research on the impact of cigarette warnings has been limited. Cross sectional evaluation studies of previous Canadian and Australian warnings suggest that warning labels are an effective means of communicating the health effects of smoking. ${ }^{3-5}$ To date, however, only one longitudinal study has examined the impact of warning labels on smoking cessation. This research found that Australian warnings introduced in 1994 prompted some smokers to delay smoking or "butt-out" a cigarette early. ${ }^{4}{ }^{6}$

New cigarette warnings labels were introduced in Canada in December 2000. These warnings have set international precedents for their size (over $50 \%$ of the package) and vivid colour images (fig 1). The warnings feature one of 16 messages on the front and back of the package, and one of 16 additional messages on the inside, which provide more detailed health risk information, as well as quit-tips and messages encouraging smokers to quit.
The present study sought to examine the impact of the new Canadian warnings on adult smoking behaviour. A measure of cognitive processing was developed to determine whether smokers who read, thought about, and discussed the labels were more likely to intend to quit smoking at baseline and to quit, make a quit attempt, or reduce their smoking at three month follow up. Finally, the study sought to assess any "wear out" or decrease in the salience of the warning labels from baseline to follow up.

\section{METHODS}

Sample

Participants were 622 adult smokers living in south western Ontario, Canada. Adult smokers were defined as individuals 18 years of age or older who had smoked at least 100 cigarettes in their lifetime and smoked at least one cigarette per day at the time of the survey. Six eligible smokers were unable to complete the survey because of cognitive or English language difficulties and were excluded from the study, leaving a final sample size of 616.

\section{Procedure}

Telephone calls were made to randomly selected telephone numbers from a list of households in South-Western Ontario, using a modified Mitofsky-Waksburg technique. ${ }^{7}$ The "most recent birthday" method ${ }^{8}$ was used to select participants from households that included more than one eligible individual. Each telephone number was attempted 12 times, at different times of the day on both weekdays and weekends before being classified as no answer. A total of 5348 numbers were tried. After eliminating ineligible numbers including businesses and non-working numbers $(\mathrm{n}=1101)$ and households not containing an eligible smoker ( $\mathrm{n}=3440)$, $14 \%(n=111)$ of participants refused or failed to complete the survey, and $10 \%(\mathrm{n}=80)$ were not reached, resulting in an AAPOR\#4 response rate of $76 \%(\mathrm{n}=616){ }^{9}$ 


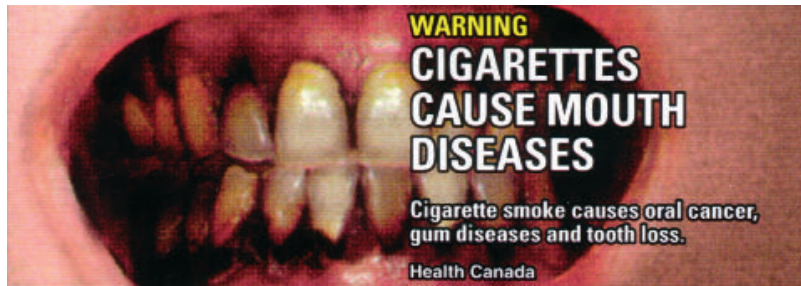

Figure 1 One of 16 health warnings on Canadian cigarette packages.

Baseline interviews were conducted by 27 trained interviewers using computer assisted telephone interviewing software (WinCATI Version 4.1, Sawtooth Software), between 9 October and 11 November 2001, approximately nine months after the introduction of the new graphic warnings. Participants were re-contacted to complete a three month follow up survey between 14 January and 26 February 2002.

The study protocol was reviewed and received ethics clearance from the University of Waterloo research ethics board.

\section{Measures}

\section{Smoking behaviour and demographic variables}

The baseline survey assessed daily cigarette consumption, number of years as a smoker, quitting history, sex, education, and age. Intention to quit smoking was measured by asking participants whether they were seriously considering quitting in the next 30 days, three months, six months, one year, or not at all.

\section{Knowledge of the warning labels}

Participants were asked to recall the location of the warning labels on cigarette packages. Participants were also asked to identify the warning messages on cigarette packages from a list of three actual and three bogus warnings messages.

Depth of cognitive processing of the warning labels Cognitive processing - or the extent to which information is processed or elaborated upon-is the most important determinant of memory and attitude change in response to new information. ${ }^{10} \mathrm{~A}$ measure of cognitive processing was created for the current study to assess the salience of the warnings and the extent to which smokers elaborated upon their information. Depth of processing was assessed by nine items, including the extent to which smokers attended to the warnings (for example, "How carefully have you ever read the messages on the outside of packages?"), or reflected and elaborated on the warnings (for example, "How often have you thought about the warnings on the outside of packages?"). More distal measures of cognitive processing were also included, such as talking about the warnings with others or thinking about the labels when they were out of sight. Responses were given on a five point Likert scale ("not at all/never, once, sometimes, often, all the time/a lot") and summed to create an index of depth of processing (Cronbach's $\alpha=0.83$ ).

\section{Follow up survey}

Baseline measures of smoking status, knowledge of the warning labels, and depth of cognitive processing were reassessed at three month follow up. Participants were also asked to report changes in their smoking behaviour, including changes in daily cigarette consumption and quit attempts (any attempt to quit smoking that lasted at least 24 hours). Cessation was defined as at least seven days of continuous abstinence at the time of follow up. The order of the questions for both the baseline and follow up surveys was randomised across participants.

\section{Statistical analysis}

Logistic regression analyses were conducted to predict quitting, quit attempts, and reductions in smoking during the three month follow up. A reduction in smoking was defined as a decrease of at least one cigarette per day. A dichotomous variable was also created to predict an overall index of cessation related behaviour, where $0=$ no cessation behaviour and 1 = participants who had either quit, made at least one quit attempt, or had reduced their smoking. Finally, regression analyses did not adjust for age given its near perfect correlation with years smoking. All analyses were conducted using SPSS software (version 10.0).

\section{RESULTS}

\section{Characteristics of sample}

A total of 616 participants completed the baseline survey. Table 1 shows that the characteristics of the study participants were similar to a representative sample of Canadian smokers from the Canadian Tobacco Monitoring Use Survey. ${ }^{11}$ The one exception is that a greater proportion of study participants were female; however, sex was not associated with any of the predictors in the regression analyses, presented below.

A total of 432 participants completed the three month follow up survey, for a follow up rate of $70.1 \%$. There were no significant differences between completers and non-completers on any measure of smoking status or demographic variables.

\section{Knowledge and depth of cognitive processing of the warning labels}

Participants demonstrated a strong knowledge of the warning labels, particularly for the warnings on the outside of packages. For example, $70.3 \%$ of participants correctly recognised three actual and three bogus messages, while only $3.6 \%$ were unable to identify a single warning. Table 2 indicates that the depth of cognitive processing for the warnings was generally high. In particular, $93.1 \%$ had read the outside warnings at least once, while $44.4 \%$ had read the

\begin{tabular}{|c|c|c|}
\hline Variable & Sample $(n=616)$ & Canada \\
\hline $\begin{array}{l}\text { Sex (females) } \\
\text { Education (minimum of } 12 \text { years) }\end{array}$ & $\begin{array}{l}56.8 \% \\
52.1 \%\end{array}$ & $\begin{array}{l}46.6 \% \dagger \\
51.3 \%\end{array}$ \\
\hline Mean age & 39.0 & 40.2 \\
\hline Cigarettes per day & 16.2 & 17.0 \\
\hline Years smoking & 20.7 & 21.4 \\
\hline Prior quit attempts & 3.5 & - \\
\hline Intentions to quit (within 6 months) & $41.2 \%$ & $42.5 \%$ \\
\hline
\end{tabular}

*Data from the Canadian Tobacco Use Monitoring Survey. ${ }^{10}$ $\mathrm{tp}<0.05$. 
Table 2 Depth of cognitive processing of the Canadian warning labels

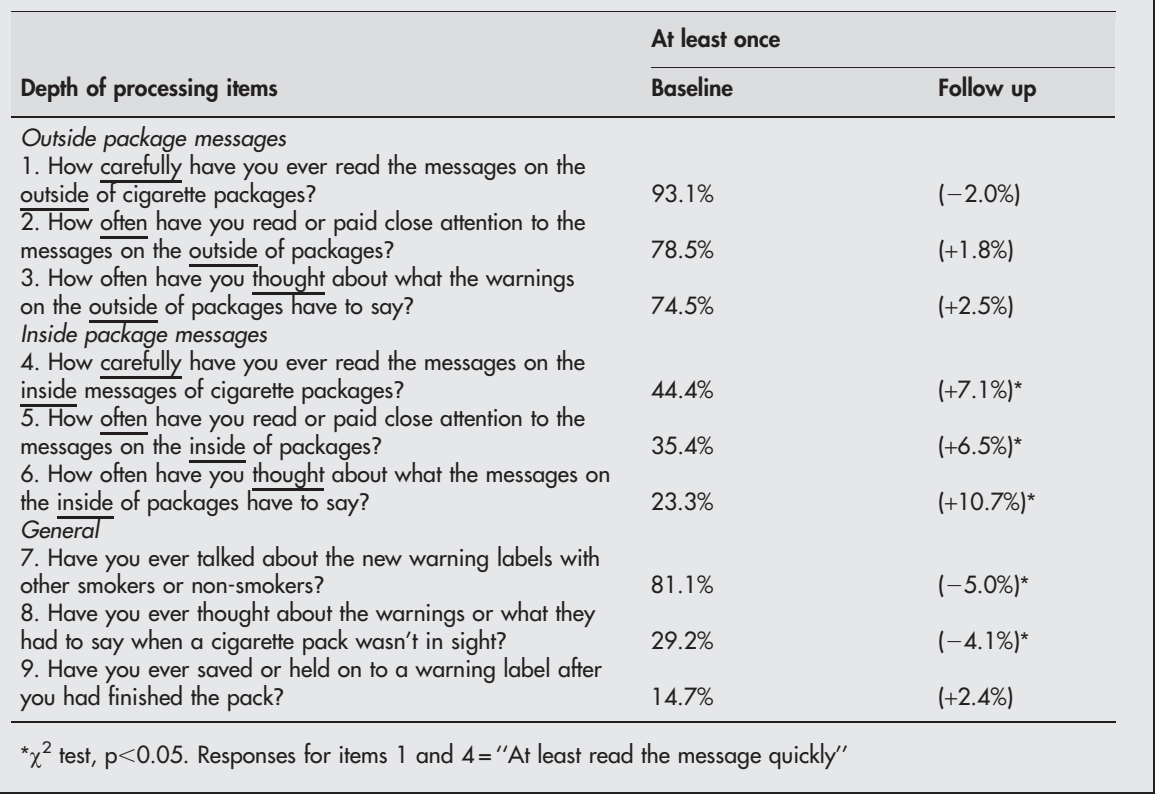

inside warnings at least once. Overall cognitive processing of the warnings decreased from baseline to follow up $(-0.27$ standard deviations, $\mathrm{p}<0.001)$. Despite this general decrease, smokers were more likely to read and think about the messages on the inside of packages at follow up (table 2).

\section{Intentions to quit at baseline}

At baseline, $41.2 \%$ of participants intended to quit smoking within the next six months. A logistic regression was conducted to examine whether cognitive processing of the warnings was associated with intentions to quit. Smokers who read, thought about, and discussed the warnings labels in greater depth at baseline were indeed more likely to intend to quit in the next six months, after adjusting for smoking status at baseline (intention to quit, daily consumption, years smoking, and prior quit attempts), sex and educational status (odds ratio (OR) 1.11, 95\% confidence interval (CI) 1.07 to 1.16; $\mathrm{p}<0.001)$. Figure 2 depicts the dose-response relation between quartile scores of cognitive processing and intentions to quit.

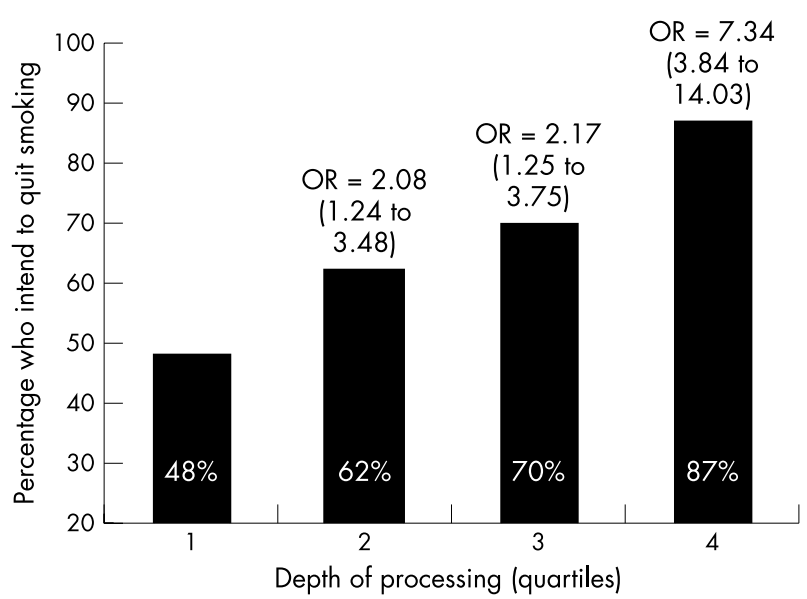

Figure 2 Intention to quit as a function of depth of cognitive processing of the warning labels at baseline.

\section{Cessation behaviour at follow up}

A total of $23.1 \%(n=100)$ participants made an attempt to quit smoking during the three month follow up period. Of these, $40(10.8 \%$ of the total sample) remained abstinent at follow up, while $24.3 \%(n=105)$ had reduced their daily cigarette consumption. Figure 3 shows the relation between depth of processing at baseline and individual changes in smoking behaviour at three month follow up. A logistic regression was also conducted to determine whether depth of processing scores at baseline could predict which smokers had engaged in any cessation behaviour. As table 3 indicates, smokers who read, thought about, and discussed the warnings labels in greater depth at baseline were significantly more likely to either quit, attempt to quit, or reduce their smoking at follow up, adjusting for smoking status at baseline (intention to quit, daily consumption, years smoking, and prior quit attempts), and sex and educational status. Figure 4 depicts the relation between quartile scores of depth of processing and cessation behaviours.

\section{DISCUSSION}

This research provides the most comprehensive evaluation of cigarette warning labels and smoking behaviour to date. In general, the vivid features of the Canadian warnings have succeeded in engaging smokers. Virtually all participants reported making some effort to read the warnings and demonstrated a strong knowledge of their basic features. Reading and thinking about the warnings was also found to be positively associated with intentions to quit. Although the direction of this relationship is unclear-smokers who intend to quit may be more likely to read the labels-the relationship is most likely reciprocal in nature. Most important, the graphic warnings appear to serve as an effective cessation intervention, as cognitive processing of the labels predicted future cessation behaviour: smokers who read, discussed, and thought about the warnings at baseline were more likely to have quit, made a quit attempt, or have reduced their smoking at three month follow up. Overall, these findings are consistent with previous research on cognitive processing and cigarette warning labels, ${ }^{12}{ }^{13}$ as well as the only other field study of cigarette warnings. ${ }^{6}$

The effectiveness of warning labels has been found to erode over time as smokers become desensitised to their 


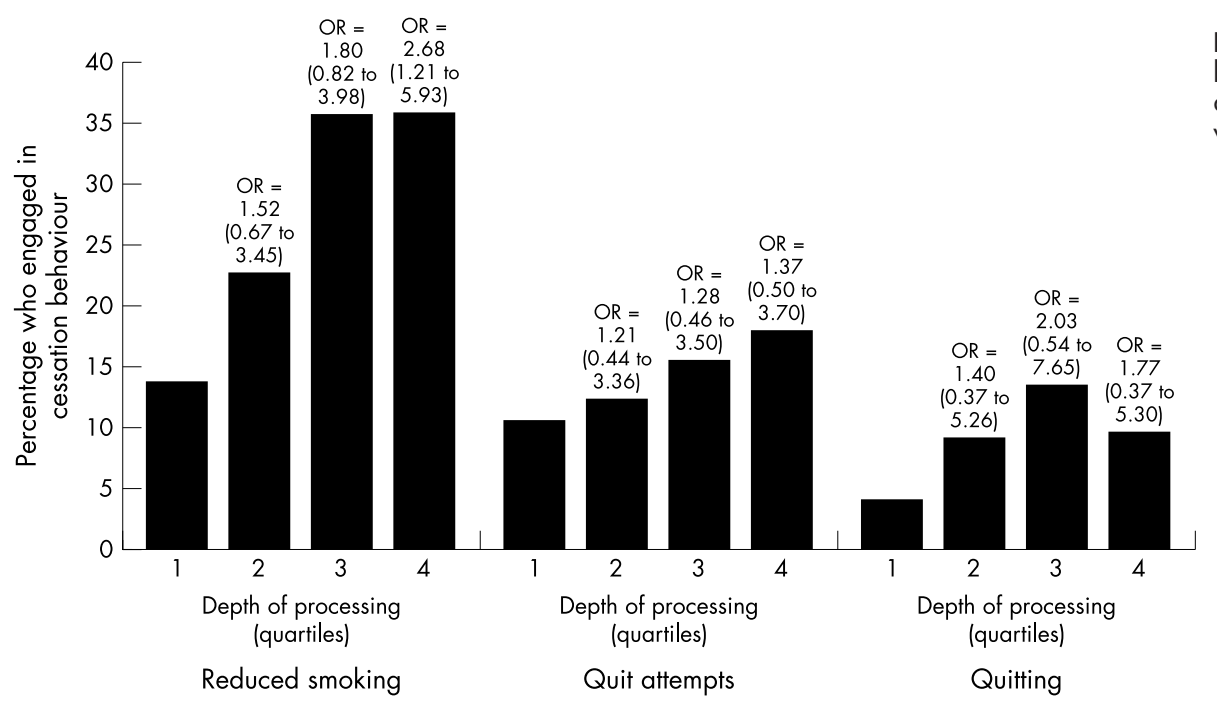

Figure 3 Individual cessation behaviours at follow up as a function of depth of cognitive processing of the warning labels at baseline.

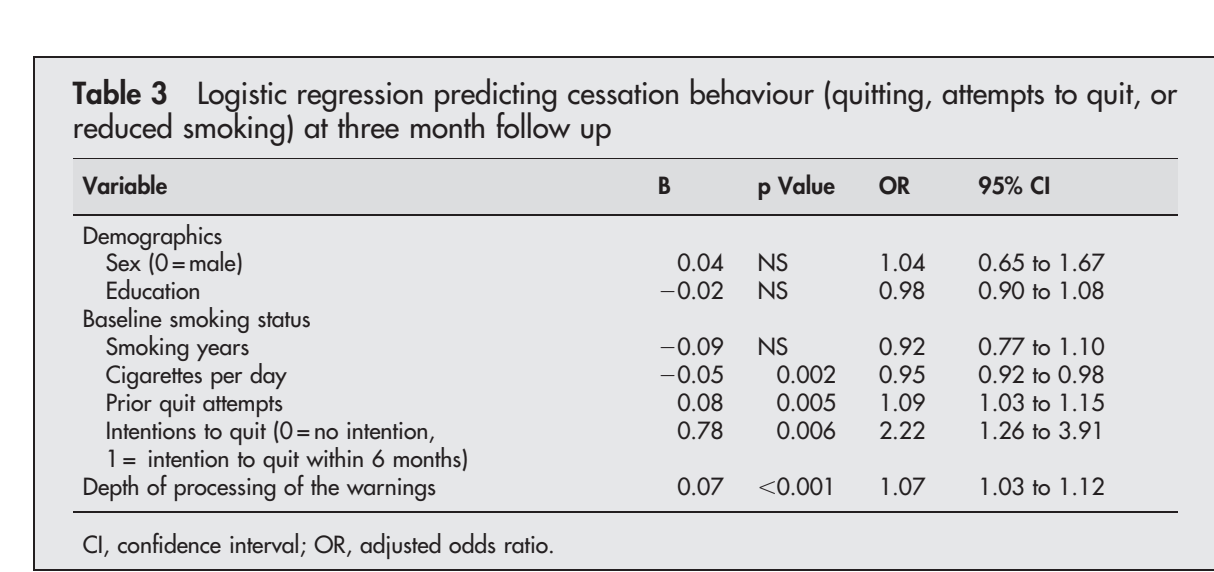

messages. ${ }^{3}$ The current findings, however, indicate very little erosion or wear out in the salience of the Canadian warning labels between 9-12 months after their introduction. Although talking about the warnings and reading the outside messages decreased as expected, in many cases processing of the warnings remained the same and the salience of the messages on the inside of packages actually increased from baseline to follow up. That the labels remained salient to smokers more than a year after they were introduced is likely a function of the number of different warnings, the amount of information they provide on the inside and outside of packages, and the variety of information provided, including cessation advice and information on the benefits of quitting. These results are consistent with smokers' own evaluations of the warning labels: smokers were more likely to report that the warnings had caused them to smoke fewer cigarettes or think about the health risks more often 12 months after their introduction, than they were after nine months. ${ }^{14}$

The study has several limitations. First, analyses predicting individual cessation behaviours suffered from a lack of power. As a result, many of the odds ratios for individual cessation behaviours did not reach significance. Nevertheless, all of the odds ratios were in the expected direction and the findings depict a consistent dose-response relation between cognitive processing and four different cessation related measures: intentions to quit, quitting, attempts to quit, and reductions in smoking. A longer follow up would allow for a greater proportion of cessation behaviours and additional power for these analyses. A longer follow up period would also provide further insight into any erosion in the impact of cigarette warning labels over time. To date, there is little evidence available to policymakers on either the timeline or extent of this decrease. Finally, this study can only speak to the impact of the warning labels on adult smokers. However, a recent quasi-experimental study has found that the Canadian cigarette warnings have enhanced label salience, perceived effectiveness, and intentions to quit among Canadian youth compared to US youth, over a similar time span as the present study. ${ }^{15}$

There is a recent trend towards increasingly vivid warning labels in tobacco labelling policy. Brazil has recently introduced colour pictures on cigarette packages and the

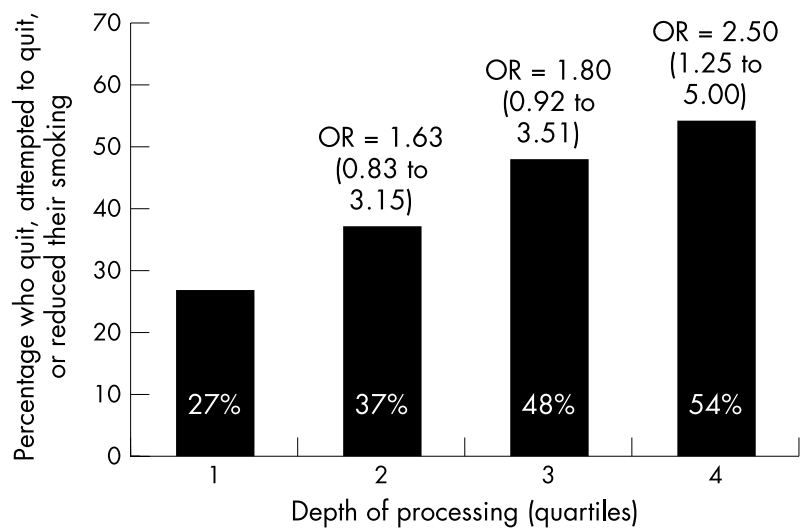

Figure 4 Cessation behaviour at follow up as a function of depth of cognitive processing of the warning labels at baseline. 


\section{What this paper adds}

Previous research indicates that cigarette warning labels are an effective means of communicating the health risks of smoking; however, there is a lack of research that examines the effect of cigarette warning labels on smoking behaviour.

This longitudinal study suggests that graphic warnings on Canadian cigarette packages may serve as an effective smoking cessation intervention for adult smokers. Virtually all smokers had read the warnings at least once and smokers demonstrated a strong knowledge of their content. Most important, smokers who read, discussed, and thought about the warnings at baseline were more likely to have quit, made a quit attempt, or reduced their smoking at three month follow up. The findings provide strong support for Canada's tobacco labelling policy and for the effectiveness of graphic cigarette warning labels.

UK is one of several European jurisdictions considering adopting "Canadian style" warning labels. Perhaps most important, the Framework Convention on Tobacco Control endorses the need for larger warnings that include pictures. There is a need for research to guide these policy developments. To this end, the current findings indicate that graphic warnings labels are a salient means of communicating health risk information and may serve as an effective smoking cessation intervention.

\section{ACKNOWLEDGEMENTS}

This research was supported by the National Cancer Institute of Canada (NCIC) with funds from the Canadian Cancer Society (CCS) and the CCS/NCIC Centre for Behavioural Research and Program Evaluation, the Ontario Tobacco Research Unit, and by a grant from the US National Cancer Institute (ROI CA 90955). We gratefully acknowledge the assistance of the Survey Research Centre and the Health Behaviour Research Group at the University of Waterloo.

\section{Authors' affiliations}

D Hammond, Department of Psychology, University of Waterloo, Waterloo, Ontario, Canada

P W McDonald", R Cameront, Department of Health Studies, University of Waterloo
G T Fongł, K S Brown§, Ontario Tobacco Research Unit, Toronto, Ontario, Canada

*Also Ontario Tobacco Research Unit, and Centre for Behavioural Research and Program Evaluation, Canadian Cancer Society/National Cancer Institute of Canada

†Also Centre for Behavioural Research and Program Evaluation, Canadian Cancer Society/National Cancer Institute of Canada $\ddagger$ Also Centre for Behavioural Research and Program Evaluation, Canadian Cancer Society/National Cancer Institute of Canada, and Department of Psychology, University of Waterloo

$\S$ Also Centre for Behavioural Research and Program Evaluation,

Canadian Cancer Society/National Cancer Institute of Canada, and

Department of Statistics and Actuarial Science, University of Waterloo

\section{REFERENCES}

1 Jah P, Chaloupka FJ, eds. Curbing the epidemic: governments and the economics of tobacco control. Washington DC: World Bank, 1999.

2 Strahan EJ, Fabrigar LR, Fong GT, et al. Enhancing the effectiveness of message labels on tobacco packaging: a social psychological perspective. Tobacco Control 2002;11:183-90.

3 Tandemar Research. Cigarette packaging study: the evaluation of new health warning messages. Report for Health Canada. Ottawa, Ontario: Health Canada, 1996.

4 Environics Research Group. Baseline surveys: The health effects of tobacco and health warning messages on cigarette packages. Report for Health Canada. Ottawa, Ontario: Health Canada, 2001.

5 Hill D. New cigarette-packet warnings: are they getting through? Med J Aust 1988; 148:478-80.

6 Borland R. Tobacco health warnings and smoking-related cognitions and behaviours. Addiction 1997;92:1427-35.

7 Waksberg J. Sampling methods for random digit dialing. J Am Statistical Assoc 1978;73:40-6.

8 O'Rourke D, Blair J. Improving random respondent selection in telephone surveys. Journal of Marketing Research 1983;20:428-32.

9 American Association for Public Opinion Research. Best practices for survey and public opinion research and survey practices that AAPOR condemns. AAPOR, 2001

10 Anderson, JR. Cognitive psychology and its implications, 3rd ed. New York: WH Freedman, 1990.

11 Health Canada. Trends in smoking 2000. Canadian Tobacco Use Monitoring Survey (CTUMS). Ottawa, Ontario: Health Canada, 2001.

12 Krugman DM, Fox RJ, Fletcher JE, et al. Do adolescents attend to warnings in cigarette advertising? An eye tracking approach. Journal of Advertising Research 1994;Nov/Dec:39-52

13 Fischer PM, Richards EJB, Krugman DM. Recall and eye tracking study of adolescents viewing tobacco advertisements. JAMA 1989;261:84-9.

14 Hammond D, Fong GT, McDonald PW, et al. Graphic cigarette warning labels do not lead to adverse outcomes: evidence from Canadian smokers. Am $J$ Public Health (in press).

15 Fong GT, Cameron AJR, Brown KS, et al. Effects of the Canadian graphic warning labels among high school students: a quasi-experimental longitudinal survey. Paper presented at the 2002 National Conference on Tobacco or Health: San Francisco, California, 2002. 